\title{
Objectively Measured Physical Activity and Health-related Quality of Life as Predictors of Mortality in U.S. Adults
}

\author{
Peter D. Hart ${ }^{*}$ \\ Health Promotion Research, Havre, MT 59501 \\ *Corresponding author: pdhart@outlook.com
}

Received November 02, 2019; Revised December 10, 2019; Accepted December 15, 2019

\begin{abstract}
Background: Few national-level studies have examined the effects of both physical activity (PA) and health-related quality of life (HRQOL) on mortality. The purpose of this study was to examine the ability of PA and HRQOL to predict all-cause mortality in U.S. adults. Methods: Data for this research came from the 2003-2004 National Health and Nutrition Examination Survey (NHANES) and its 2011 mortality-linked National Death Index (NDI) file. Moderate-to-vigorous PA (MVPA, min/day) was objectively determined by accelerometry and participants were categorized into low or high groups using the median. HRQOL was assessed by a single question asking participants to rate their general health. Participants rating their health as "good", "very good", or "excellent" were considered to have good HRQOL whereas those rating it "fair" or "poor" were considered to have poor HRQOL. Cox proportional hazard regression was used to determine the independent effects of MVPA and HRQOL on all-cause mortality while controlling for covariates. Results: In the fully adjusted females model, participants were at greater risk of mortality if they engaged in low amounts of MVPA (Hazard Ratio (HR) $=2.82,95 \%$ CI: $1.02-$ 7.85) and had poor HRQOL (HR=7.19, 95\% CI: 2.30-22.51) with no significant ( $p=.133$ ) MVPA-by-HRQOL interaction. Conversely, the equivalent males model saw a significant $(p=.002)$ MVPA-by-HRQOL interaction, resulting in an analysis of simple effects. In the fully adjusted males with good HRQOL model, participants were at greater risk of mortality if they engaged in low amounts of MVPA (HR=2.92, 95\% CI: 1.56-5.49). However, in the males with poor HRQOL model, MVPA could not significantly predict mortality (HR=1.04, 95\% CI: 0.49-2.24). Conclusion: Results from this study indicate that MVPA and HRQOL independently predict all-cause mortality in U.S. female adults. In U.S. male adults, MVPA predicts all-cause mortality only in those with good HRQOL.
\end{abstract}

Keywords: physical activity, Accelerometry, Health-related quality of life (HRQOL), Mortality

Cite This Article: Peter D. Hart, "Objectively Measured Physical Activity and Health-related Quality of Life as Predictors of Mortality in U.S. Adults.” American Journal of Public Health Research, vol. 7, no. 6 (2019): 197-202. doi: 10.12691/ajphr-7-6-2.

\section{Introduction}

Physical activity (PA) is a universally known health behavior promoted to all adults for its positive health benefits including increased health-related quality of life (HRQOL) [1-5]. Despite this recommendation, the continual collection of data supporting the relationship between PA and health outcomes is important so as to improve the body of knowledge. This is especially true when a large portion of the current knowledge has come from research utilizing self-report assessments of PA, which may lack the same precision seen with objective assessments [6]. Additionally, many studies supporting the positive role of PA on health outcomes have used samples of convenience, which potentially bias certain generalizations [7]. Lastly, HRQOL is most commonly used as a patient-reported outcome and less so as a predictor variable for survival studies $[8,9,10]$. Therefore, the purpose of this study was to examine the concurrent ability of objectively measured PA and HRQOL to predict all-cause mortality in a representative sample of U.S. adults.

\section{Methods}

\subsection{Participants and Design}

Data for this research came from the 2003-2004 National Health and Nutrition Examination Survey (NHANES) and associated mortality file [11,12]. Mortality data were linked to NHANES participants by the National Center for Health Statistics (NCHS) and for this study the mortality status follow-up ended December 31, 2011. Participants who were 20+ years of age, had complete PA data, and were eligible for mortality linkage were used in the analysis. 


\subsection{Measures}

Moderate-to-vigorous PA (MVPA, min/day) was objectively determined by use of accelerometry [13] and participants were categorized into low and high groups using the median. Additionally, participants were categorized into MVPA quartiles where lower quartiles contained less-active participants. HRQOL was assessed by a single question asking participants to rate their general health [14]. In this study, participants rating their health as "good", "very good", or "excellent" were considered to have good HRQOL whereas those rating it "fair" or "poor" were considered to have poor HRQOL. Covariates were used to describe the sample and control confounding in the statistical models. These covariates included sex (male/female), age (yr), race/ethnicity (White/Black/Hispanic/Other), and income (U.S. \$).

\subsection{Statistical Analysis}

Prevalence estimates with their standard errors (SEs) were computed for HRQOL overall and across demographic groups. Means and their SEs were computed for MVPA overall and across demographic groups. Differences between sex groups were tested using chi-square tests of independence (HRQOL), logistic regression (HRQOL), and ordinary least squares regression (MVPA). Cox proportional hazards regression was employed separately for each sex group to model the effects of MVPA and HRQOL on mortality while controlling for age, race, and income. Finally, MVPA-by-HRQOL interactions were tested in adjusted models - and if found significant - resulted in simple effects modeling. SAS version 9.4 survey procedures were used to account for the sampling design [15-18]. All significance levels were set to $p=.05$.

\section{Results}

A total of 5,031 adults were initially included in the survival analysis with a mean (median) person-year follow-up of 7.4 (7.8) years and 726 assumed deaths by all causes. Table 1 contains baseline mean estimates and tests of mean differences by sex for continuous values of MVPA. Males (Mean MVPA=30.4 min/day) overall engaged in significantly $(p<.05)$ more baseline MVPA than females (Mean MVPA=16.7 min/day). This same pattern was also significantly $(p s<.05)$ consistent across all demographic groupings. Table 2 displays baseline prevalence estimates and tests of proportion differences by sex for poor HRQOL. There was a suggestive tendency for more females (18.8\%) to report poor HRQOL at baseline as than males $(15.8 \%, p=.70)$. With significantly more females reporting poor HRQOL than males if they were 18 to 24 years of age $(p=.008)$, Black $(p<.001)$, or had income less than $\$ 25,000$ per year $(p s<.05)$.

Table 1. Baseline moderate-to-vigorous physical activity (MVPA, min/day) across demographic characteristics and by sex, US adults 20+ years of age 2003-2004

\begin{tabular}{|c|c|c|c|c|c|c|c|}
\hline \multirow[b]{2}{*}{ Characteristics } & \multicolumn{2}{|c|}{ Overall } & \multicolumn{2}{|c|}{ Males } & \multicolumn{2}{|c|}{ Females } & \multirow{2}{*}{$\frac{\text { Sex Diff }}{p}$} \\
\hline & Mean & $S E$ & Mean & $S E$ & Mean & $S E$ & \\
\hline Overall $(N=3,080)$ & 23.3 & 0.89 & 30.4 & 0.98 & 16.7 & 0.74 & $<.001$ \\
\hline \multicolumn{8}{|l|}{ Age Group (yr) } \\
\hline $20-24$ & 34.0 & 2.14 & 44.5 & 4.16 & 24.9 & 1.28 & $<.001$ \\
\hline $25-34$ & 31.6 & 1.34 & 39.6 & 1.61 & 22.5 & 1.78 & $<.001$ \\
\hline $35-44$ & 30.0 & 1.58 & 39.1 & 1.45 & 20.7 & 1.36 & $<.001$ \\
\hline $45-54$ & 24.1 & 0.89 & 30.9 & 1.26 & 17.4 & 0.90 & $<.001$ \\
\hline $55-64$ & 17.0 & 0.94 & 20.7 & 1.24 & 14.2 & 1.29 & .003 \\
\hline $65+$ & 8.5 & 0.60 & 10.5 & 0.83 & 6.9 & 0.66 & .001 \\
\hline \multicolumn{8}{|l|}{ Race/Ethnicity } \\
\hline White & 22.2 & 0.85 & 28.6 & 0.99 & 16.1 & 0.70 & $<.001$ \\
\hline Black & 23.6 & 1.33 & 32.2 & 1.90 & 16.6 & 1.85 & $<.001$ \\
\hline Hispanic & 32.1 & 1.86 & 42.1 & 2.59 & 21.9 & 2.30 & $<.001$ \\
\hline Other & 21.2 & 2.62 & 27.4 & 3.64 & 16.2 & 2.62 & .006 \\
\hline \multicolumn{8}{|l|}{ Annual Income (\$) } \\
\hline $0-14,999$ & 17.1 & 1.71 & 25.5 & 2.34 & 11.2 & 1.37 & $<.001$ \\
\hline $15,000-24,999$ & 18.4 & 1.65 & 25.2 & 2.83 & 13.6 & 1.47 & $<.001$ \\
\hline $25,000-44,999$ & 22.2 & 1.35 & 29.6 & 1.67 & 15.7 & 1.13 & $<.001$ \\
\hline $45,000-54,999$ & 23.7 & 1.48 & 29.9 & 2.22 & 17.6 & 1.36 & $<.001$ \\
\hline $65,000-74,999$ & 25.6 & 2.33 & 34.4 & 3.39 & 14.8 & 1.45 & $<.001$ \\
\hline $75,000+$ & 28.7 & 0.82 & 34.0 & 1.06 & 22.6 & 0.76 & $<.001$ \\
\hline
\end{tabular}

Note. MVPA reported as means (se) and associated hypothesis tests are from ordinary least squares regression. Sex Diff p-values are testing for proportion differences between sex groups. 
Table 2. Baseline health-related quality of life (HRQOL) across demographic characteristics and by sex, US adults 20+ years of age 2003-2004

\begin{tabular}{|c|c|c|c|c|c|c|c|}
\hline \multirow[b]{2}{*}{ Characteristics } & \multicolumn{2}{|c|}{ Poor HRQOL: Overall } & \multicolumn{2}{|c|}{ Poor HRQOL: Males } & \multicolumn{2}{|c|}{ Poor HRQOL: Females } & \multirow{2}{*}{$\frac{\text { Sex Diff }}{p}$} \\
\hline & $\%$ & $S E$ & $\%$ & $S E$ & $\%$ & $S E$ & \\
\hline Overall $(N=4,320)$ & 17.3 & 1.18 & 15.8 & 1.21 & 18.8 & 1.65 & .070 \\
\hline \multicolumn{8}{|l|}{ Age Group (yr) } \\
\hline $18-24$ & 9.8 & 1.38 & 5.0 & 1.98 & 14.5 & 2.01 & .008 \\
\hline $25-34$ & 10.1 & 1.53 & 10.4 & 1.46 & 9.9 & 2.20 & .824 \\
\hline $35-44$ & 15.9 & 2.04 & 15.9 & 2.56 & 15.9 & 2.68 & .996 \\
\hline $45-54$ & 18.8 & 1.64 & 17.5 & 2.20 & 20.1 & 2.72 & .476 \\
\hline $55-64$ & 20.1 & 2.40 & 19.0 & 3.57 & 21.0 & 2.36 & .574 \\
\hline $65^{+}$ & 27.1 & 2.45 & 24.3 & 1.41 & 29.4 & 3.85 & .130 \\
\hline \multicolumn{8}{|l|}{ Race/Ethnicity } \\
\hline White & 15.1 & 1.68 & 14.7 & 1.81 & 15.5 & 2.00 & .645 \\
\hline Black & 24.4 & 1.19 & 17.9 & 1.42 & 29.5 & 1.85 & $<.001$ \\
\hline Hispanic & 26.8 & 1.36 & 23.7 & 2.64 & 30.0 & 2.72 & .173 \\
\hline Other & 13.5 & 3.19 & 8.4 & 4.02 & 18.3 & 5.66 & .195 \\
\hline \multicolumn{8}{|c|}{ Annual Income (US \$) } \\
\hline $0-14,999$ & 34.9 & 1.88 & 30.9 & 1.77 & 37.5 & 2.75 & .029 \\
\hline $15,000-24,999$ & 28.6 & 2.61 & 24.7 & 2.41 & 31.5 & 3.35 & .016 \\
\hline $25,000-44,999$ & 18.0 & 1.82 & 19.0 & 1.94 & 17.2 & 2.61 & .545 \\
\hline $45,000-54,999$ & 13.1 & 1.69 & 14.2 & 3.20 & 12.0 & 2.12 & .596 \\
\hline $65,000-74,999$ & 10.2 & 2.34 & 7.1 & 2.34 & 14.2 & 4.28 & .110 \\
\hline $75,000+$ & 7.8 & 1.06 & 8.5 & 1.60 & 7.0 & 1.37 & .479 \\
\hline
\end{tabular}

Note. HRQOL reported as percentages (se) and associated hypothesis tests are chi-square tests of independence or logistic regression. Sex Diff p-values are testing for proportion differences between sex groups.

Table 3. Hazards of all-cause mortality associated with moderate-to-vigorous physical activity (MVPA, high/low) and health-related quality of life (HRQOL, good/poor), US adults 20+ years of age, baseline (2003-2004) thru follow-up (2011)

\begin{tabular}{|c|c|c|c|c|c|c|c|c|c|}
\hline \multirow[b]{2}{*}{ Grouping } & \multicolumn{3}{|c|}{ Crude } & \multicolumn{3}{|c|}{ Model 1} & \multicolumn{3}{|c|}{ Model 2} \\
\hline & HR & $95 \%$ CI & $p$ & HR & $95 \%$ CI & $\mathrm{p}$ & HR & $95 \% \mathrm{CI}$ & $\mathrm{p}$ \\
\hline \multicolumn{10}{|l|}{ Females } \\
\hline \multicolumn{10}{|l|}{ MVPA } \\
\hline High & 1.00 & - & & 1.00 & - & & 1.00 & - & \\
\hline Low & 6.31 & $2.95-13.49$ & $<.001$ & 6.55 & $2.29-18.78$ & .002 & 2.82 & $1.02-7.85$ & .047 \\
\hline \multicolumn{10}{|l|}{ HRQOL } \\
\hline Good & 1.00 & - & & 1.00 & - & & 1.00 & - & \\
\hline Poor & 4.87 & $3.65-6.49$ & $<.001$ & 8.54 & $2.31-31.57$ & .003 & 7.19 & $2.30-22.51$ & .002 \\
\hline MVPA*HRQOL & & & & & & .225 & & & .133 \\
\hline \multicolumn{10}{|l|}{ Males } \\
\hline \multicolumn{10}{|l|}{ MVPA } \\
\hline High & 1.00 & - & & 1.00 & - & & 1.00 & - & \\
\hline Low & 6.52 & $3.81-11.17$ & $<.001$ & 7.99 & $4.11-15.52$ & $<.001$ & 3.36 & $1.78-6.33$ & .001 \\
\hline \multicolumn{10}{|l|}{ HRQOL } \\
\hline Good & 1.00 & - & & 1.00 & - & & 1.00 & - & \\
\hline Poor & 3.86 & $3.08-4.84$ & $<.001$ & 8.54 & $3.60-20.26$ & $<.001$ & 6.95 & $3.37-14.32$ & $<.001$ \\
\hline MVPA*HRQOL & & & & & & .010 & & & .002 \\
\hline
\end{tabular}

Note. Crude column shows unadjusted HRs for MVPA and HRQOL in separate models. Model 1 column shows unadjusted HRs for both MVPA and HRQOL in same model. Model 2 column shows HRs for MVPA and HRQOL adjusted for age, race, and income. Cox regression was used for all models using PROC SURVEYPHREG to account for the complex sampling design. 
Table 4. Hazards of all-cause mortality associated with moderate-to-vigorous physical activity (MVPA, high/low) and health-related quality of life (HRQOL, good/poor), US males 20+ years of age, baseline (2003-2004) thru follow-up (2011)

\begin{tabular}{|c|c|c|c|c|c|c|}
\hline \multirow[b]{2}{*}{ Grouping } & \multicolumn{3}{|c|}{ Crude } & \multicolumn{3}{|c|}{ Model 1} \\
\hline & HR & $95 \%$ CI & $\mathrm{p}$ & HR & $95 \%$ CI & $\mathrm{p}$ \\
\hline \multicolumn{7}{|c|}{ Males with poor health } \\
\hline \multicolumn{7}{|l|}{ MVPA } \\
\hline High & 1.00 & - & & 1.00 & - & \\
\hline Low & 2.34 & $1.18-4.63$ & .018 & 1.04 & $0.49-2.24$ & .907 \\
\hline \multicolumn{7}{|c|}{ Males with good health } \\
\hline \multicolumn{7}{|l|}{ MVPA } \\
\hline High & 1.00 & - & & 1.00 & - & \\
\hline Low & 7.99 & $4.12-15.50$ & $<.001$ & 2.92 & $1.56-5.49$ & $<.001$ \\
\hline \multicolumn{7}{|c|}{ Males with low MVPA } \\
\hline \multicolumn{7}{|l|}{ HRQOL } \\
\hline Good & 1.00 & - & & 1.00 & - & \\
\hline Poor & 2.56 & $2.08-3.16$ & $<.001$ & 2.11 & $1.67-2.66$ & $<.001$ \\
\hline \multicolumn{7}{|c|}{ Males with high MVPA } \\
\hline \multicolumn{7}{|l|}{ HRQOL } \\
\hline Good & 1.00 & - & & 1.00 & - & \\
\hline Poor & 8.67 & $3.58-21.00$ & $<.001$ & 5.87 & $2.34-14.70$ & $<.001$ \\
\hline
\end{tabular}

Note. Crude column shows unadjusted HRs for MVPA and HRQOL in separate models. Model 1 column shows HRs for MVPA and HRQOL adjusted for age, race, and income. Cox regression was used for all models using PROC SURVEYPHREG to account for the complex sampling design.

Table 3 displays sex-specific hazards of all-cause mortality associated with MVPA (high/low) and HRQOL (good/poor) for both crude and adjusted analyses. In the fully adjusted females model (adjusted for age, race, and income), females were at greater risk of mortality if they engaged in low amounts of MVPA (Hazard Ratio $(\mathrm{HR})=2.82$, 95\% CI: 1.02-7.85) and had poor HRQOL (HR=7.19, 95\% CI: 2.30-22.51) with no significant $(p=.133)$ MVPA-by-HRQOL interaction. Conversely, the equivalent males model saw a significant ( $p=.002)$ MVPA-by-HRQOL interaction. Table 4 displays the resulting analysis of simple effects for males. In the fully adjusted males with good HRQOL model, males were at greater risk of mortality if they engaged in low amounts of MVPA (HR=2.92, 95\% CI: 1.56-5.49). However, in the males with poor HRQOL model, MVPA could not significantly predict mortality $(\mathrm{HR}=1.04,95 \% \mathrm{CI}: 0.49$ 2.24).

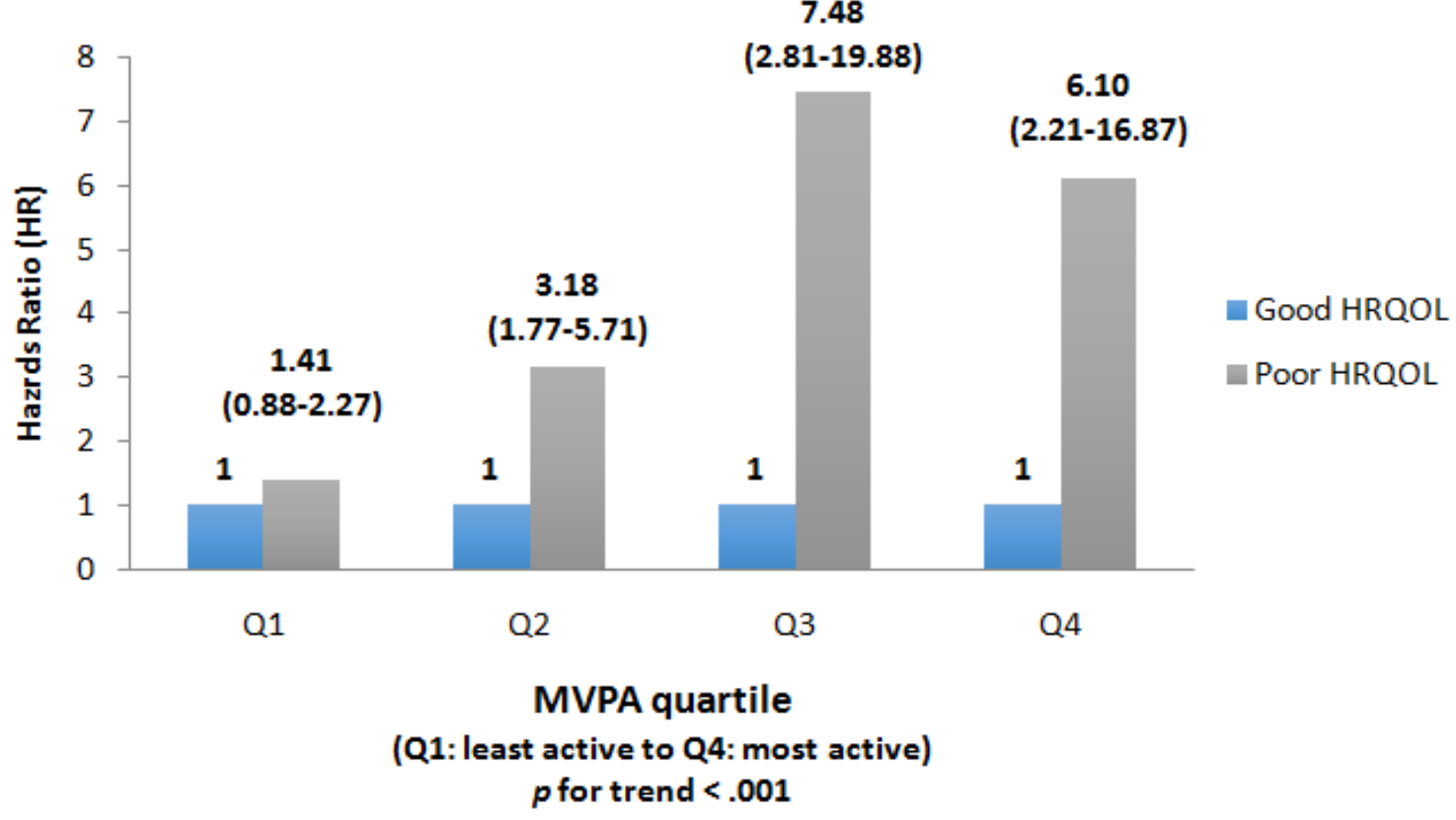

Figure 1. Males (Age-adjusted hazards of all-cause mortality associated with poor HRQOL by MVPA quartile) 


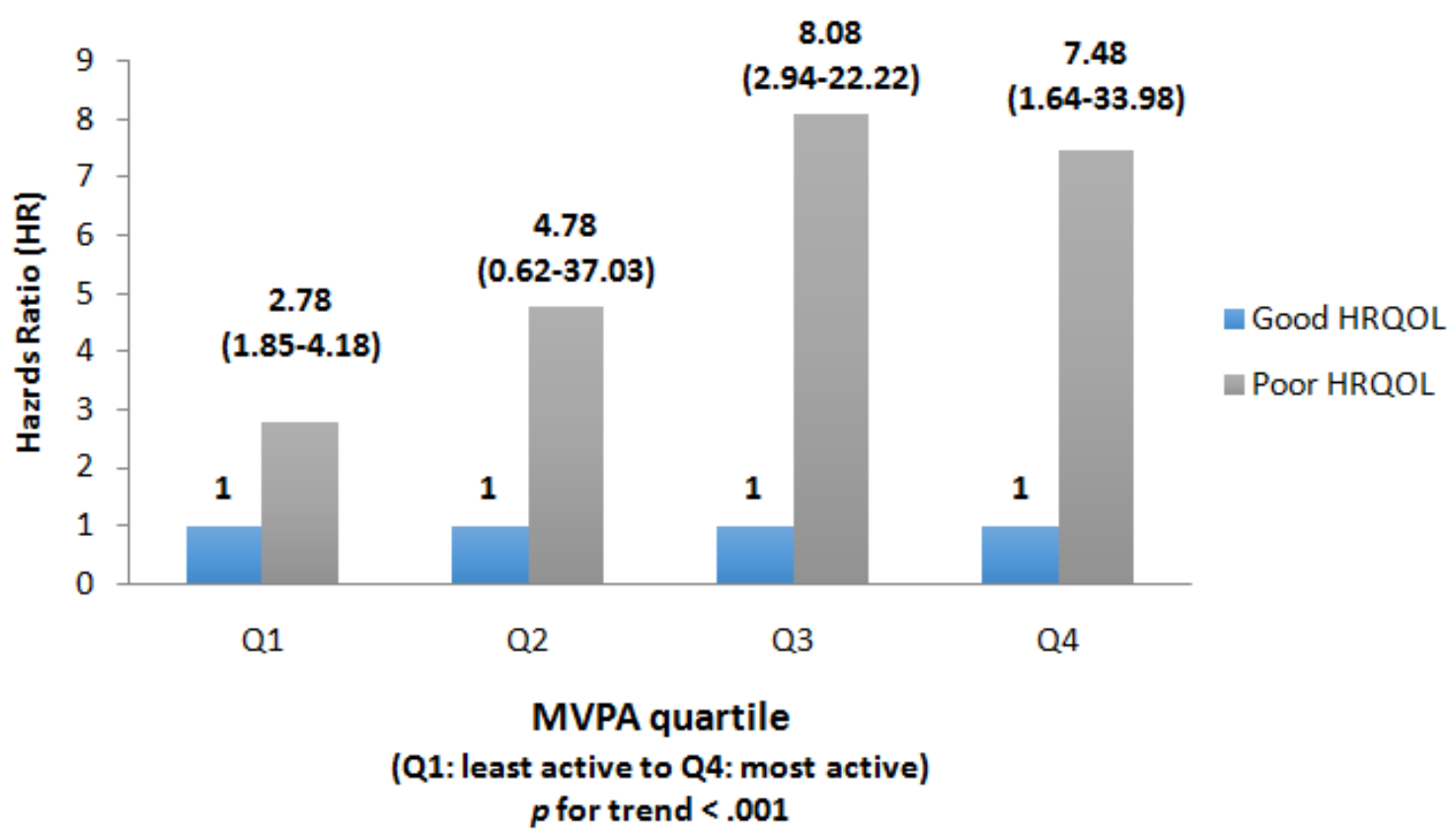

Figure 2. Females (Age-adjusted hazards of all-cause mortality associated with poor HRQOL by MVPA quartile)

Figure 1 and Figure 2 show the HRs (95\% CIs) associated with HRQOL status across MVPA quartiles. Both graphs indicate a similar trend of increased risk of mortality among those who report poor HRQOL (as compared to those who report good HRQOL). However, the mortality risk associated with poor HRQOL tended to increase as MVPA increased. This linear trend ( $p$ s for trend $<.001$ ) was similar for both sex groups.

\section{Discussion}

This research reports several noteworthy findings. Firstly, MVPA and HRQOL were shown to independently predict all-cause mortality in the female analysis. Furthermore, the independent association of both predictors withstood the model adjustment for age, race, and income. This finding indicates that regardless of a women's HRQOL status, participation in MVPA is likely to increase her longevity. Similarly, regardless of a women's MVPA status, improving her HRQOL to "good" status is likely to increase her longevity. Secondly, and conversely, MVPA and HRQOL were not found to independently predict all-cause mortality in the male analysis. What this part of the results revealed is that HRQOL is a necessary predictor of all-cause mortality in adult males. That is, regardless of a males MVPA status, poor HRQOL predicts mortality. However, only among adult males with good HRQOL, can MVPA predict mortality. This finding implicates HRQOL as a more important protective factor than PA for adult males. Thirdly, and lastly, when considering MVPA in ordinal groupings of quartiles, it appears that poor HRQOL strength as a predictor of mortality increased as the amounts of MVPA increased. This relationship was noticed in both sex-specific analyses with significant linear trends. These findings also highlight the possibility that large amounts of MVPA may not protect against the mortality-predictive power of poor HRQOL.
A strength of this study is that it examined the concurrent impact of two major risk factors (PA and HRQOL) on mortality and examined their independence as predictors. Therefore, this study adds to the science above those studies that examine only a single risk factor. Another strength of this study is its use of a nationally representative sample of adults 20+ years of age. Hence, this study has an advantage over similar studies that examine relationships between PA, HRQOL and mortality in specific subpopulations or in samples of convenience.

This study also has at least one weakness - which may be the use of self-reported general health to assess HRQOL. Therefore, this study must accept the possibility of some HRQOL misclassification due to the subjective nature of the assessment. Given this shortcoming, HRQOL is a perceived concept that is typically measured by self-report [19]. Additionally, the single general health item used in this study has been validated and assumed to be a reliable measure of HRQOL [20]. Regardless, because of this limitation, findings from this study should be interpreted with caution.

\section{Conclusions}

Results from this study indicate that MVPA and HRQOL independently predict all-cause mortality in U.S. female adults. In U.S. male adults, MVPA predicts all-cause mortality only in those with good HRQOL. Health promotion advocates promoting PA should focus equally on improving HRQOL when longevity is of concern, especially among men.

\section{Acknowledgements}

No financial assistance was used to assist with this project. 


\section{References}

[1] Physical Activity Guidelines Advisory Committee. Physical activity guidelines advisory committee report, 2008. Washington, DC: US Department of Health and Human Services. 2008 Jun 24; 2008: A1-H14.

[2] US Department of Health and Human Services, Office of Disease Prevention and Health Promotion, US Department of Health and Human Services, Office of Disease Prevention and Health Promotion. Healthy people 2020.

[3] Hart, P. D., Benavidez, G. A., \& Erickson, J. (2017). Meeting recommended levels of physical activity in relation to preventive health behavior and health status among adults. Journal of Preventive Medicine and Public Health. 2017; 50(1): 10-17.

[4] Hart PD, Buck DJ. The effect of resistance training on healthrelated quality of life in older adults: Systematic review and metaanalysis. Health promotion perspectives. 2019; 9(1): 1.

[5] Hart PD. Meeting recommended levels of physical activity and health-related quality of life in rural adults. Journal of lifestyle medicine. 2016 Mar; 6(1): 1.

[6] Jakicic JM, King WC, Gibbs BB, Rogers RJ, Rickman AD, Davis KK, Wahed A, Belle SH. Objective versus self-reported physical activity in overweight and obese young adults. Journal of Physical Activity and Health. 2015 Oct 1; 12(10): 1394-400.

[7] DeFina LF, Radford NB, Barlow CE, Willis BL, Leonard D, Haskell WL, Farrell SW, Pavlovic A, Abel K, Berry JD, Khera A. Association of all-cause and cardiovascular mortality with high levels of physical activity and concurrent coronary artery calcification. JAMA cardiology. 2019 Feb 1; 4(2): 174-81.

[8] Roser K, Mader L, Baenziger J, Sommer G, Kuehni CE, Michel G. Health-related quality of life in Switzerland: normative data for the SF-36v2 questionnaire. Quality of life research. 2019 Jul 15; 28(7): 1963-77.
[9] Shin WK, Song S, Jung SY, Lee E, Kim Z, Moon HG, Noh DY, Lee JE. The association between physical activity and healthrelated quality of life among breast cancer survivors. Health and quality of life outcomes. 2017 Dec; 15(1): 132.

[10] Vallance JK, Boyle T, Courneya KS, Lynch BM. Associations of objectively assessed physical activity and sedentary time with health-related quality of life among colon cancer survivors. Cancer 2014 Sep 15; 120(18): 2919-26.

[11] Zipf G, Chiappa M, Porter KS, et al. National Health and Nutrition Examination Survey: Plan and operations, 1999-2010. National Center for Health Statistics. Vital Health Stat 1(56). 2013.

[12] National Center for Health Statistics. Office of Analysis and Epidemiology. Analytic Guidelines for NCHS 2011 Linked Mortality Files, August, 2013. Hyattsville, Maryland

[13] Centers for Disease Control and Prevention National Center for Health Statistics. NHANES 2003-2004 Laboratory Procedures Manual: 16. Physical Activity Monitor; 2004.

[14] Centers for Disease Control and Prevention National Center for Health Statistics. NHANES 2003-2004 Current Health Status HSQ; 2004.

[15] Tabachnick BG, Fidell LS. Using multivariate statistics, 5th. Needham Height, MA: Allyn \& Bacon. 2007.

[16] Cody RP, Smith JK. Applied statistics and the SAS programming language. North-Holland; 1985.

[17] Allison PD. Survival analysis using SAS: a practical guide. SAS Institute; 2010.

[18] SAS/STAT(R) 14.1 User's Guide. Introduction to Survey Sampling and Analysis Procedures. SAS Institute. July 2015.

[19] Hennessy CH, Moriarty DG, Zack MM, Scherr PA, Brackbill R Measuring health-related quality of life for public health surveillance. Public health reports. 1994 Sep; 109(5): 665.

[20] Cunny KA, Perri III M. Single-item vs multiple-item measures of health-related quality of life. Psychological reports. 1991 Aug; 69(1): 127-30

(C) The Author(s) 2019. This article is an open access article distributed under the terms and conditions of the Creative Commons Attribution (CC BY) license (http://creativecommons.org/licenses/by/4.0/). 